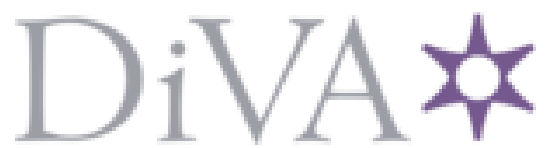

http://www.diva-portal.org

This is the published version of a paper published in Clinical Physiology and Functional Imaging.

Citation for the original published paper (version of record):

Rosendahl, L., Blomstrand, P., Heiberg, E., Ohlsson, J., Björklund, P-G. et al. (2008)

Computer-assisted calculation of myocardial infarct size shortens the evaluation time of contrast-enhanced cardiac MRI

Clinical Physiology and Functional Imaging, 28(1): 1-7

https://doi.org/10.1111/j.1475-097X.2007.00765.x

Access to the published version may require subscription.

N.B. When citing this work, cite the original published paper.

Permanent link to this version:

http://urn.kb.se/resolve?urn=urn:nbn:se:oru:diva-72076 


\title{
Computer-assisted calculation of myocardial infarct size shortens the evaluation time of contrast-enhanced cardiac MRI
}

\author{
Lene Rosendahl ${ }^{1,2}$, Peter Blomstrand ${ }^{1}$, Einar Heiberg ${ }^{3}$, Jan Ohlsson ${ }^{1}$, Per-Gunnar Björklund ${ }^{1}$, Britt-Marie \\ Ahlander ${ }^{4}$ and Jan Engvall ${ }^{2,5}$ \\ ${ }^{1}$ Department of Clinical Physiology, County Hospital Ryhov, Jönköping, ${ }^{2}$ Center for Medical Image Science and Visualization, CMIV, Linköping, ${ }^{3}$ Department \\ of Clinical Physiology, Lund University Hospital, Lund, ${ }^{4}$ Department of Radiology, County Hospital Ryhov, Jönköping, and ${ }^{5}$ Department of Clinical Physiology, \\ Linköping University Hospital, Linköping, Sweden
}

\section{Summary}

\section{Correspondence}

Lene Rosendahl, Department of Clinical Physiology, County Hospital Ryhov, 55185 Jönköping, Sweden E-mail: lene.rosendahl@lj.se

\section{Accepted for publication}

Received 24 April 2007;

accepted 20 September 2007

\section{Key words}

computer software; magnetic resonance imaging; myocardial infarct size;

semi-automatic; time saving
Background: Delayed enhancement magnetic resonance imaging depicts scar in the left ventricle which can be quantitatively measured. Manual segmentation and scar determination is time consuming. The purpose of this study was to evaluate a software for infarct quantification, to compare with manual scar determination, and to measure the time saved.

Methods: Delayed enhancement magnetic resonance imaging was performed in 40 patients where myocardial perfusion single photon emission computed tomography imaging showed irreversible uptake reduction suggesting a myocardial scar. After segmentation, the semi-automatic software was applied. A scar area was displayed, which could be corrected and compared with manual delineation. The different time steps were recorded with both methods.

Results: The software shortened the average evaluation time by $12.4 \mathrm{~min}$ per cardiac exam, compared with manual delineation. There was good correlation of myocardial volume, infarct volume and infarct percentage (\%) between the two methods, $r=0.95, r=0.92$ and $r=0.91$ respectively.

Conclusion: A computer software for myocardial volume and infarct size determination cut the evaluation time by more than 50\% compared with manual assessment, with maintained clinical accuracy.

\section{Introduction}

The prognosis after a myocardial infarction is strongly related with the infarct size (Moss, 1983; Miller et al., 1995; Wu et al., 1998). Small infarcts, $<18 \%$ of the left ventricular myocardium, have a good prognosis (Wu et al., 1998). Myocardial perfusion single photon emission computed tomography imaging (MPS) provides an automatic calculation of cardiac volume and perfusion defects, but the method exposes the patient to radiation and is sensitive to attenuation effects. Cardiac magnetic resonance imaging (MRI) has undergone great progress and its ability to assess patients with various heart diseases is constantly increasing. After the injection of a gadolinium-based contrast agent, Delayed enhancement magnetic resonance imaging (DE-MRI) accurately visualizes regional myocardial necrosis in ischaemic heart disease (Kim et al., 1999). Because of the high spatial resolution, DE-MRI has the ability to assess the transmurality of a myocardial infarct, which is important for (C) 2007 The Authors

Journal compilation (c) 2007 Blackwell Publishing Ltd • Clinical Physiology and Functional Imaging 28, 1, 1-7 differentiating between viable and non-viable myocardium (Kim et al., 2000; Choi et al., 2001; Selvanayagam et al., 2004). Currently DE-MRI is considered to be the method of choice for assessing myocardial infarct volume, superior to both the positron emission tomography technique and MPS for detecting subendocardial scar (Klein et al., 2002; Wagner et al., 2003).

Manual delineation of myocardial scar is time consuming and there is a need to develop image analysis methods that accurately quantify DE-MRI images. Visual estimation of the global myocardial extent of hyperenhancement (Comte et al., 2004) as well as semi-quantitative visual scoring compared with planimetry, has been suggested (Azevedo Filho et al., 2004). Objective semi-automatic methods for the analysis of myocardial infarct size have been described (Hsu et al., 2006a,b) while, to the best of our knowledge, only Positano et al. (2005) reported the time necessary for performing the measurements.

Heiberg et al. (2005) presented a freely available computer software, Segment, (http://segment.heiberg.se) that showed 
good agreement in the determination of infarct size compared with a consensus of three manual observers. Semi-automatic quantification showed $6 \cdot 1 \pm 6 \cdot 6 \mathrm{ml}$ (mean $\pm \mathrm{SD}$ ) larger scar than the mean of the three observers with an interobserver variability (SD) of $4 \cdot 2 \mathrm{ml}$. The same segmentation of myocardial borders was used for the manual as well as for the semiautomatic calculation. The time saving with the semi-automatic method was not reported. Therefore, in the present study we measured the time necessary for applying the semi-automatic computer algorithm for infarct size quantification compared with manual delineation and estimated if the agreement between the two methods was within clinically acceptable limits.

\section{Material and methods}

\section{Study population}

Forty patients, 33 men and seven women, age $65 \pm 10$ years (range: 36-84) were consecutively enrolled between June 2002 and March 2004. Patients referred for MPS on suspicion of coronary artery disease were included if they had an irreversible uptake reduction suggesting a myocardial scar or if an attenuation defect was deemed improbable. A reversible component on MPS was allowed. Exclusion criteria were contraindications for MRI such as an implantable cardiac device, ferromagnetic or intracranial clips, claustrophobia or an intercurrent cardiovascular event between the studies, such as revascularization or myocardial infarction. No patient was excluded because of technical failure or poor image quality. MPS and MRI were performed within $42 \pm 34$ days (range 10-192). The study was approved by the Ethics committee at Linköping University and complied with the Declaration of Helsinki. All patients gave informed consent.

\section{Magnetic resonance imaging}

The patients were placed in the magnet (1. $5 \mathrm{~T}$ MagnetomVision; Siemens, Erlangen, Germany) in a supine position. A circular polarized body-array surface coil was used in all measurements. ECG-triggered MR images were obtained during repeated breath-holds. For cine imaging, a turbo-fast low angle shot (FLASH)-sequence was used. The contrast-enhanced images were acquired at the same positions as the cine-images. Gadopentetate dimeglumine (Gd-DTPA) $0.2 \mathrm{mmol} \mathrm{kg}^{-1}$ bodyweight was administered intravenously in 33 patients and $0 \cdot 1 \mathrm{mmol} \mathrm{kg}{ }^{-1}$ bodyweight in seven patients. A segmented IR turbo-FLASH-sequence was used, with a repetition time determined by 2 R-R intervals, an echo time of $3.4 \mathrm{~ms}$ and an inversion time of 175-250 ms with $300 \mathrm{~ms}$ delay after the Rwave. Slice thickness was $8 \mathrm{~mm}$ and intersection gap $2 \mathrm{~mm}$. To cover the entire left ventricle, an average of nine slices was needed (range 7-11). Field-of-view was $270 \times 360 \mathrm{~mm}$ and image matrix $132 \times 256 \mathrm{~mm}$. The segmented sequence acquired $33 \mathrm{k}$-space lines following the inversion pulse. A
$300 \mathrm{~ms}$ delay forced the data acquisition to the diastolic phase. The sequence was repeated every other heartbeat four times. Total acquisition time per slice was 10 heartbeats including one magnetization steady-state preparation period. Optimal contrast between hyper-enhanced areas and normal myocardium was established by continually adjusting the inversion time, to null the signal from the healthy myocardium. The contrast-to-noise ratio of the scar area was on average $6 \cdot 8 \pm 3 \cdot 3$.

\section{Measurement of infarct size with the semi-automatic method}

Segmentation of the endo- and epicardial borders on short-axis slices was performed manually and independently by two observers, followed by running the software for automatic determination of scar volume. The papillary muscles were included in the left ventricular size/infarction size, if they were attached to the myocardium at that particular slice. The time required for segmentation was recorded. The automatic scar analysis took only a few seconds. The same automated infarct delineation algorithm as described by Heiberg et al. (2005) was used. The algorithm can be summarized as: in each slice, the mean signal intensity and SD was calculated in five sectors. The sector with the lowest mean signal intensity was considered 'remote' myocardium. A slice specific threshold was calculated as the mean of the 'remote' sector $+2 \cdot 4 \mathrm{SD}$ from the mean signal intensity in the 'remote' region. The number of SD from 'remote' was chosen after an optimization process to minimize the variability of the algorithm. A three-dimensional image processing algorithm was applied to limit the heterogeneity of the hyper-enhanced regions, and to exclude small regions that constitute noise rather than infarction. The result is shown into two steps, 'semi-automatic infarct sizing' and 'semi-automatic corrected infarct sizing'.

Based on the semi-automatic infarct sizing, if the observer did not agree with the result of the infarct area determined by the software, a manual correction was performed. Long-axis views aided the determination of infarcted myocardium. The two observers recorded the time needed for correction and the mean time of the observers was calculated.

\section{Measurement with the manual method}

Manual segmentation and infarct sizing was performed by planimetry using short-axis slices of the heart. Long-axis views aided the determination of scar at the apex. The volumes of healthy myocardium and scar were averaged from two observers. Image analysis was performed with ImageJ 1.29X (Wayne Rasband, NIH, Bethesda, MD, USA) (http://rsb.info. nih.gov/ij/). Partial volume effects, because of thick slices e.g. in the apex and in the left ventricular outflow tract, were resolved by consensus ( $6 \%$ of all slices). The time required for the measurements was recorded. The analysis process is graphically displayed in Fig. 1. An example of semi-automatic and manual infarct sizing is shown in Fig. 2. 
Figure 1 Flow chart showing the number of exams and the average evaluation time for the semi-automatic and manual infarct sizing methods. After running the software for the determination of infarct size the observers on average corrected 25 exams.
Semi-automatic method

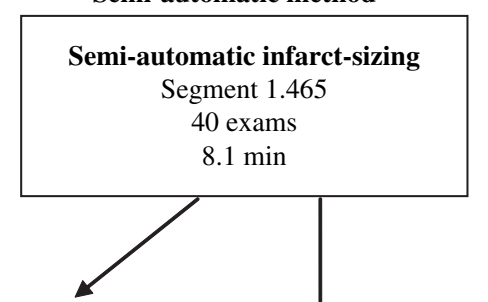

If correction needed

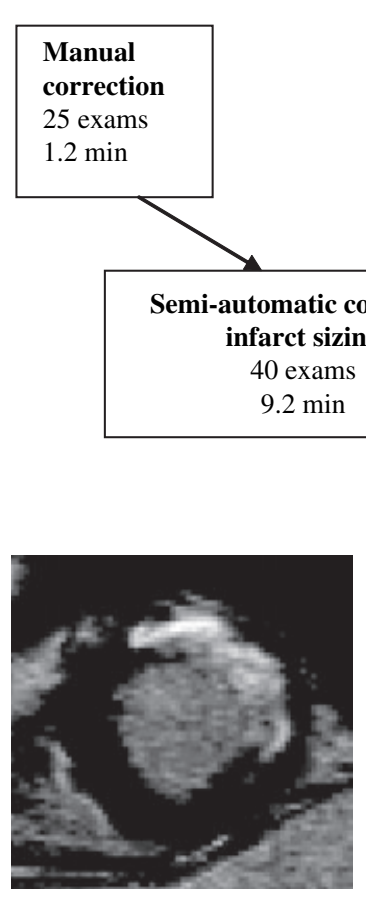

Manual method

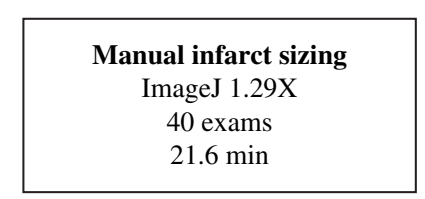

If correction not needed
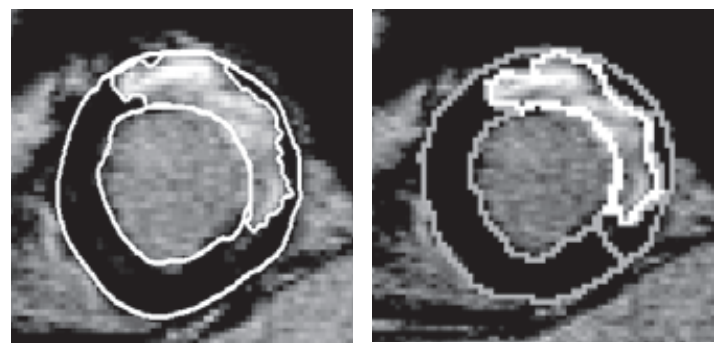

Table 1 Results, averaged from two observers, of the semi-automatic corrected method and the manual method.

Analyses were performed using sPSS 13.0 for Windows (SPSS Inc, Chicago, IL, USA). Values are reported as mean \pm SD. For myocardial volume, infarct size and infarct percentage, a twosided t-test for paired observations was used. Correlation coefficients and related P-values are reported and Bland-Altman plots used. Mean coefficient of variation (COV, \%) was calculated.

\section{Results}

The time for evaluating a cardiac MRI study was $9 \cdot 2 \pm 1 \cdot 8 \mathrm{~min}$ (range 6-14) with the semi-automatic corrected method, of which $1.2 \pm 0.6$ (range $0.5-3 \cdot 0$ ) min was devoted to minor adjustments. The manual infarct sizing required $21.6 \pm 4.5 \mathrm{~min}$ (range 15-31). The results for myocardial volume, infarct volume and infarct percentage assessed by the semi-automatic corrected and manual methods are listed in Table 1. There was no significant difference in myocardial volume between the two infarct sizing methods, Fig. 3a, Semi-automatic corrected infarct sizing showed slightly larger infarct size, $3 \cdot 8 \pm 8 \cdot 1 \mathrm{ml}$, and infarct percentage $2 \cdot 1 \pm 4 \cdot 4 \%$ than the manual method,

\begin{tabular}{lrrrl}
\hline & $\begin{array}{c}\text { Semi-automatic } \\
\text { corrected }\end{array}$ & Manual & $\boldsymbol{r}$ & Sign \\
\hline Myocardial volume (ml) & $168 \pm 51$ & $172 \pm 53$ & 0.95 & 0.09 \\
Infarct volume (ml) & $26 \pm 20$ & $22 \pm 17$ & 0.92 & 0.005 \\
Infarct percentage (\%) & $15 \pm 11$ & $13 \pm 10$ & 0.91 & 0.005 \\
\hline
\end{tabular}

$P=0.005$, Fig. 3b,c. Generally, the manual adjustments of infarct volume in the semi-automatic corrected method were small shown in Fig. 4 and Table 2.

The applied corrections were of two types. Corrections were performed if the delineation of the myocardium erroneously included parts extrinsic to the myocardium. Also, corrections were frequent if the scar area was considered to be caused by partial volume effects. There were, on average, 13 corrections of the first type and 20 corrections of the second type. For corrections of the first type, $70 \%$ were deletions and 30\% were additions. For corrections of the second type, deletions and additions were $50 \%$ each. An example of corrections is seen in Fig. 5.

(C) 2007 The Authors

Journal compilation @ 2007 Blackwell Publishing Ltd • Clinical Physiology and Functional Imaging 28, 1, 1-7 
(a)

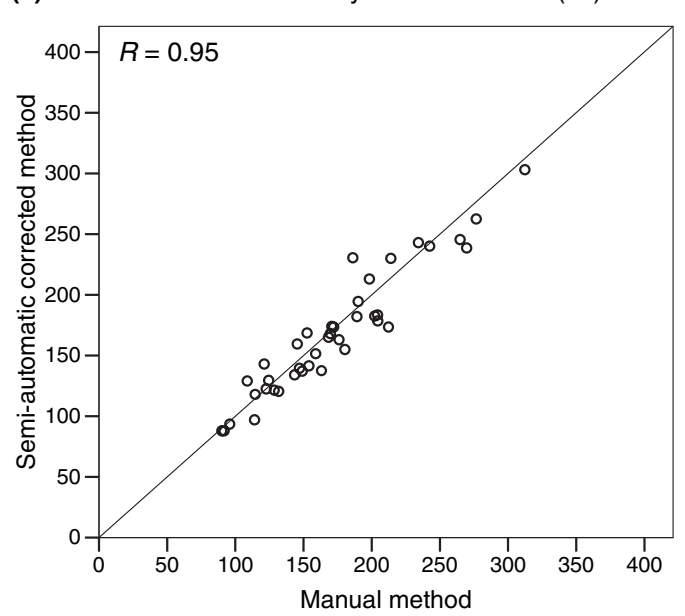

(b)

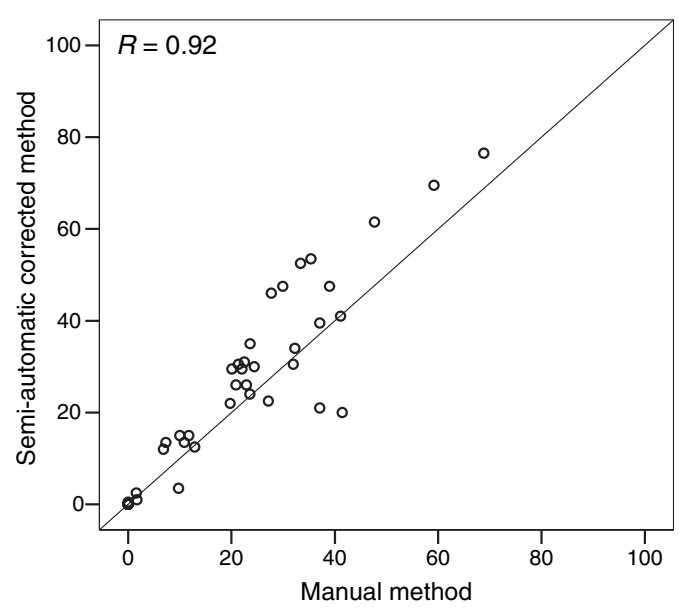

(c)

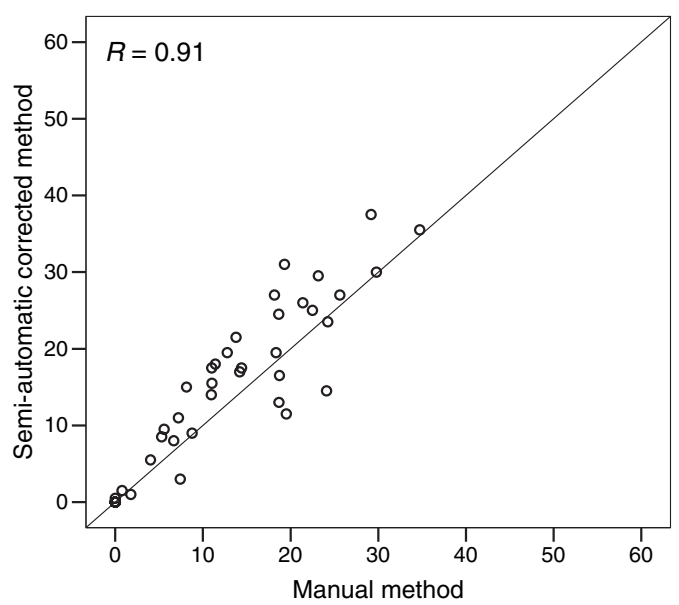

Left ventricle myocardial volume $(\mathrm{ml})$

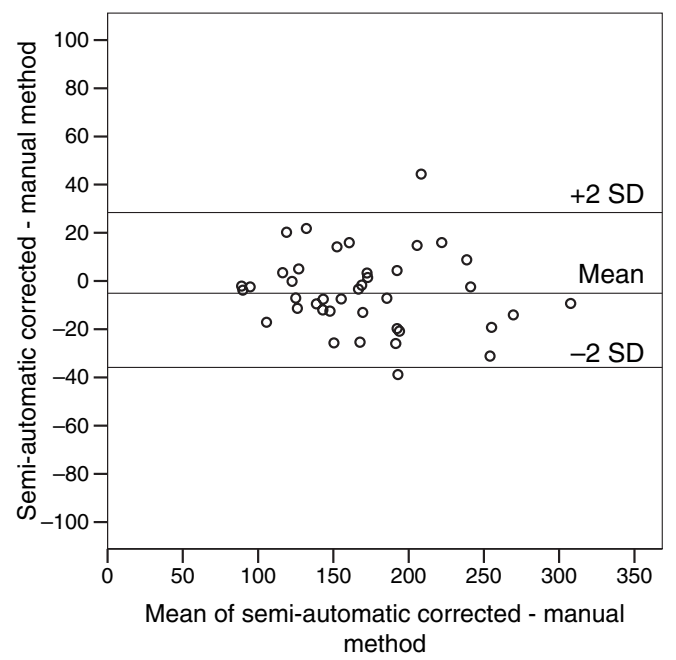

Infarct volume (ml)

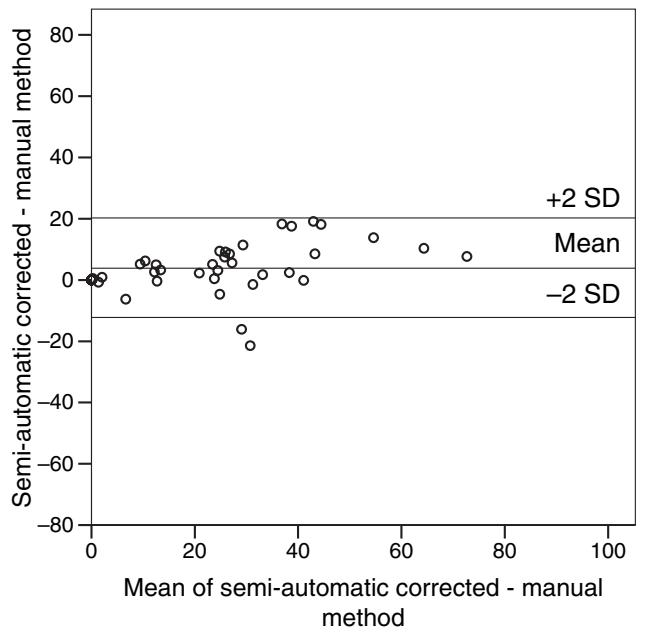

Infarct percentage

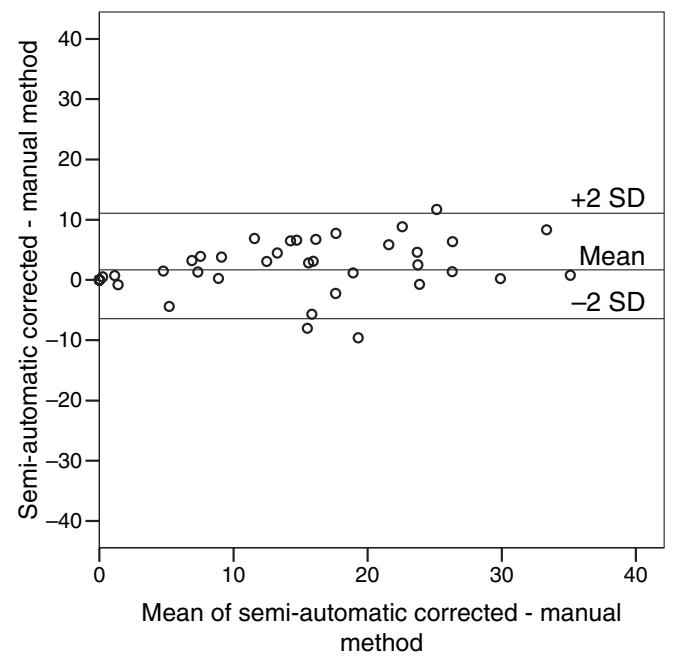

Figure 3 Values are averaged from two observers. Consensus between the observers was obtained in the manual method, but not in the semiautomatic corrected method. (a) Determination of left ventricular myocardial volume. Semi-automatic corrected versus manual method. (b) Determination of infarct volume. Semi-automatic corrected versus manual method. (c) Infarct size as a percentage of myocardial volume. Semiautomatic corrected versus manual method. 

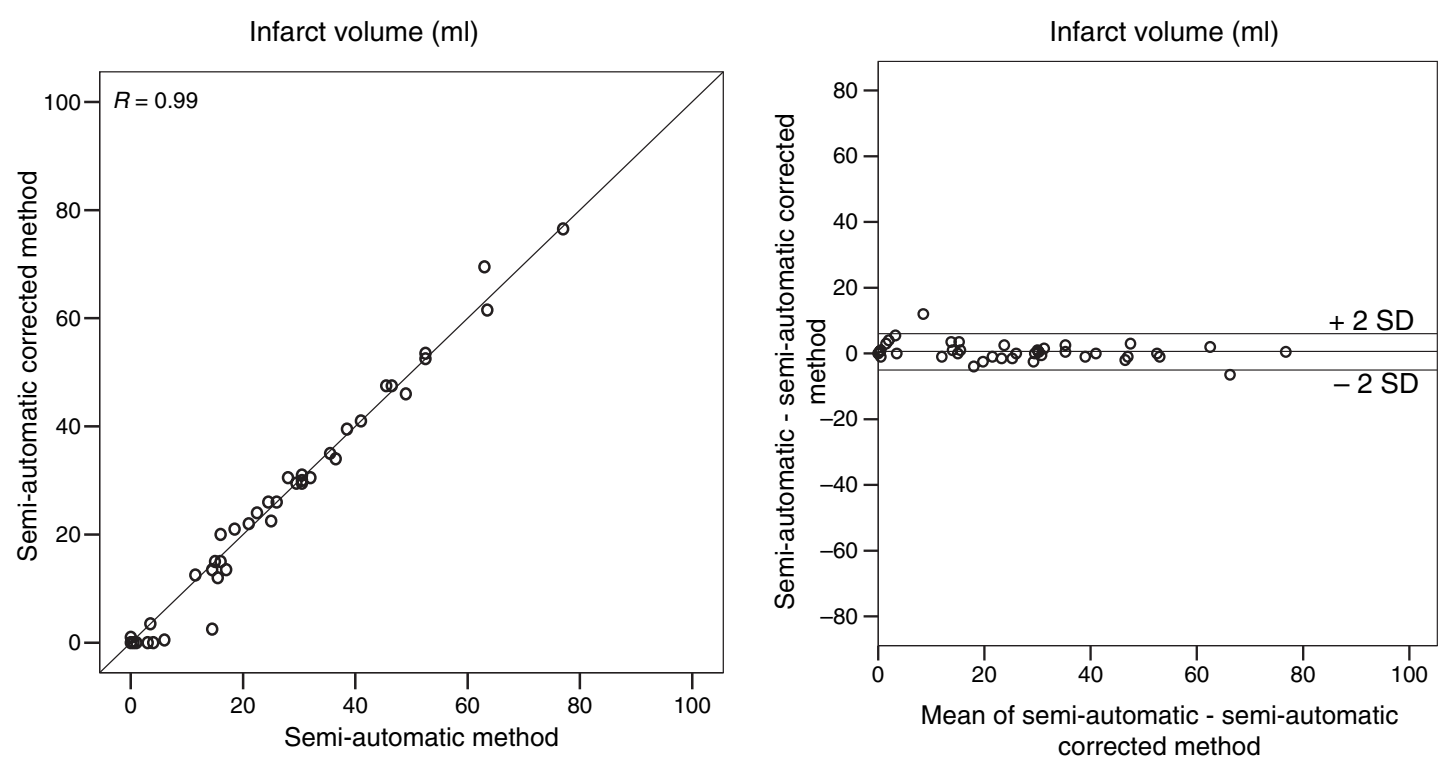

Figure 4 Values are averaged from two observers. Effect of the corrections applied to the semiautomatic infarct sizing. Corrected infarct volume (y-axis) versus software standard setting ( $x$-axis).

Table 2 Results, averaged from two observers, of performing corrections to the semi-automatic infarct sizing results. The segmentation of the myocardial volume was not changed between the measurements.

\begin{tabular}{lcccc}
\hline & & Semi-automatic & & \\
& Semi-automatic & corrected & $\boldsymbol{r}$ & Sign \\
\hline Infarct volume $(\mathrm{ml})$ & $26 \pm 19$ & $26 \pm 20$ & $0 \cdot 99$ & $0 \cdot 2$ \\
Infarct percentage (\%) & $16 \pm 10$ & $15 \pm 11$ & $0 \cdot 99$ & $0 \cdot 1$ \\
\hline
\end{tabular}

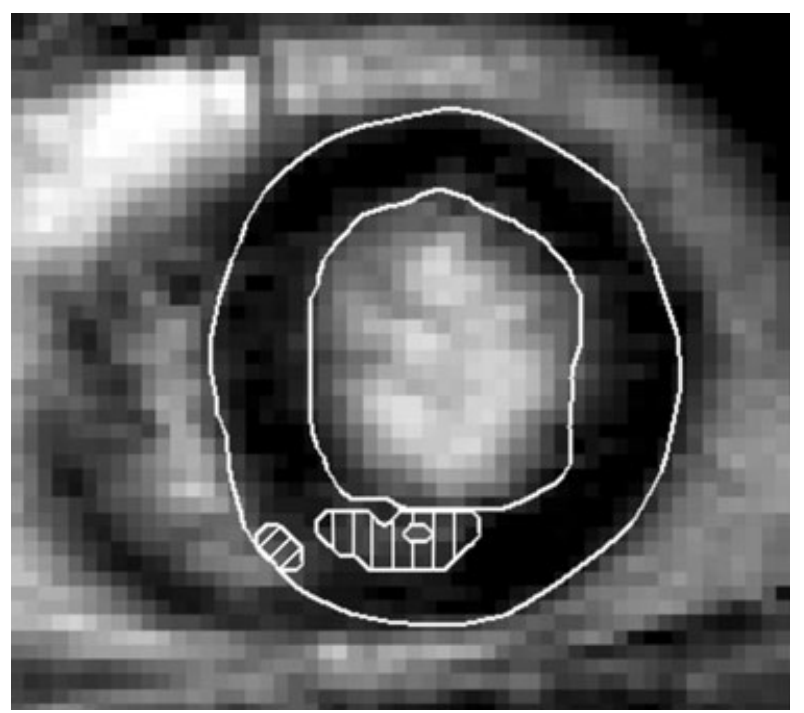

Figure 5 An example of manual corrections. $\llbracket$, area erroneously marked as infarcted by the semi-automatic method. $\mathbb{W}$, infarct area added manually. The circle marks an area correctly classified as infarcted by the semi-automatic method.

With manual infarct sizing, the intraobserver variability of the two observers for myocardial volume was $5 \cdot 2 \pm 13.6$ and $2 \cdot 4 \pm 18 \cdot 6 \mathrm{ml}$, respectively, for infarct volume $0 \cdot 3 \pm 8 \cdot 0$ and (C) 2007 The Authors Journal compilation @ 2007 Blackwell Publishing Ltd • Clinical Physiology and Functional Imaging 28, 1, 1-7
$0 \cdot 2 \pm 6 \cdot 4 \mathrm{ml}$, and for infarct percentage $0 \cdot 1 \pm 4 \cdot 3 \%$ and $0 \cdot 2 \pm 3 \cdot 8 \%$. Interobserver variability for myocardial volume was $1.7 \pm 14.7 \mathrm{ml} \quad(\mathrm{r}=0.96, \quad \mathrm{P}=0.5)$, infarct volume $1.0 \pm 3.0 \mathrm{ml}(\mathrm{r}=0.98, \quad \mathrm{P}=0.05)$ and infarct percentage $0 \cdot 3 \pm 2 \cdot 4 \%(r=0 \cdot 97, P=0 \cdot 4)$.

In the semi-automatic method, each observer used the same segmentation of the left ventricle for both semi-automatic and semi-automatic corrected infarct sizing and only adjusted the scar area. Because of different approaches to the segmentation process the interobserver variability for myocardial volume was $32.3 \pm 20.6 \mathrm{ml} \quad(\mathrm{r}=0.95, \quad \mathrm{P}=0.001), \quad$ infarct volume $11 \cdot 0 \pm 12.3 \mathrm{ml}(\mathrm{r}=0.87, \mathrm{P}=0.001)$ and infarct percentage $3 \cdot 4 \pm 5 \cdot 9 \%(r=0 \cdot 87, P=0.001)$.

Coefficient of variation was for myocardial volume $1 \%$, and for infarct volume and for infarct percentage $4 \%$ and $2 \%$, respectively, in the manual method. In the semi-automatic corrected method where consensus between the observers was not applied, COV was for myocardial volume 19\%, for infarct volume $42 \%$ and infarct percentage $22 \%$ respectively.

\section{Discussion}

Contrast-enhanced MRI has quickly established its role as the gold standard for assessing myocardial infarct size (Pennell et al., 2004). However, manual measurement is time-consuming and it is tempting to abandon it in favour of reporting a quick visual impression (Azevedo Filho et al., 2004; Comte et al., 2004). In this study, we have evaluated a computer software for the quantification of myocardial infarct size from DE-MRI. This semi-automatic program shortens, on average, the evaluation to $9 \cdot 2 \mathrm{~min}$ per patient, an average time saving of $12.4 \mathrm{~min}$ compared with the manual method. The semi-automatic method produced a slightly larger infarct volume than the 
manual method, $2 \cdot 1 \%$ points, which would not have led to misclassification of small versus larger infarcts, Fig. 3c (Wu et al., 1998).

Heiberg et al. (2005) studied the diagnostic power of this software and found good agreement compared with manual scar determination by three experienced observers. However, in a clinical work flow situation, the largest difference between observers seems to be variations in myocardial volume because of different outlining of the endo- and epicardial borders especially in difficult areas such as in the ventricular apex. This was predefined in the Heiberg study. In the present study, however, segmentation was required from each observer, thus increasing the likelihood of a spread in myocardial volume values. A partial correction for this problem is possible by forcing the observers to reach a consensus. With manual infarct sizing, consensus was needed in $6 \%$ of slices, resulting in an interobserver variation in myocardial volume of $1 \cdot 7 \pm 14.7 \mathrm{ml}$. In the semi-automatic method, when no consensus was applied, the interobserver variation for myocardial volume was $32 \cdot 3 \pm 20 \cdot 6 \mathrm{ml}$. However, even if consensus narrows the spread in values, it may not improve accuracy. This may be achieved by adding information on infarct extent from long-axis views of the cardiac apex. Partial volume effects have recently been discussed in another study of infarct size (Heiberg et al., 2007).

The DE-MRI method produces slices of the left ventricle showing bright scar areas with a high contrast compared with the dark, viable myocardium. However, the infarct border is often irregular because of the anatomic distribution of the coronary arteries as well as partial volume effects aggravated by thick myocardial slices and by the curvature of the apical parts of the left ventricle (Kim et al., 1999). In the present study, the two observers handled the partial volume effect at the cardiac apex differently, with one observer consistently delineating larger myocardial as well as scar volume, contributing to the divergence in the calculation of myocardium and infarct volume. Microvascular obstruction, which causes hypoperfused areas that can be misinterpreted as healthy myocardium, was not a problem in our study, because there were no acute infarcts in our study (Rochitte et al., 1998; Taylor et al., 2004). A particular problem of semi-automatic delineation of myocardial borders is the frequently small difference in signal intensity between the blood pool and the hyper-enhanced myocardium. A promising technique to alleviate this problem is the multicontrast delayedenhancement (MCODE) acquisition scheme, which allows co-registration of an anatomic slice together with the delayed enhancement image (Kellman et al., 2005).

Objective measurement and standardized evaluation is desirable in the application of all cardiac imaging methods. Hoffmann et al. (1996) showed that physicians employed in the same echo lab agreed well in wall motion assessment but less so when compared with physicians trained in other hospitals. A semi-automatic method for measuring scar size would facilitate the standardization of infarct assessment. Computer software does not show day-to-day variation and could serve as decision support for non-experienced observers. In a recent attempt to develop a fast and effective method for assessing myocardial scar, Positano et al. (2005) presented an algorithm that agreed well with the result of MPS with correlation $0 \cdot 79$ when assessing the global extent of necrosis. In comparison with manual assessment, their automatic segmentation and infarct determination process saved an average of $20 \cdot 7 \mathrm{~min}$.

\section{Limitations}

The time necessary for performing segmentation of the myocardium depends on the experience of the observer. In this study, it will affect both methods to the same degree. All tomographic methods for volume measurements of the left ventricle are sensitive to partial volume effects, which complicate the determination of infarct area and volume. Reducing slice thickness could have diminished partial volume effects, but at a cost of lower signal-to-noise ratio (SNR). At the time of the study, we did not have access to a three-dimensional-based sequence with its inherently higher SNR. Finally, the effect of infarct size on ventricular function is affected by the varying infarct transmurality in different segments of the left ventricle. A widely distributed subendocardial scar may cause a substantial reduction in left ventricular function despite a rather small infarct volume. This necessitates addressing transmurality as well as infarct size when evaluating the circulatory effect of a myocardial infarction.

\section{Conclusions}

The size of a myocardial infarction is of great prognostic value for the risk of developing heart failure, which in turn affects medical treatment and also the need for cardiac intervention or surgery. A computer software for myocardial volume and infarct size determination cut the evaluation time to $<50 \%$ compared with manual assessment, with maintained clinical accuracy, which enables quantification in a busy clinical work flow.

\section{Acknowledgments}

This grant support was provided by Futurum - the academy for healthcare, Jönköping County Council, CMIV at Linköping University, Linköping Heart Centre and the Swedish Heart-Lung Foundation.

\section{References}

Azevedo Filho CF, Hadlich M, Petriz JL, Mendonca LA, Moll Filho JN, Rochitte CE. Quantification of left ventricular infarcted mass on cardiac magnetic resonance imaging: comparison between planimetry and the semiquantitative visual scoring method. Arq Bras Cardiol (2004); 83: 118-124.

Choi KM, Kim RJ, Gubernikoff G, Vargas JD, Parker M, Judd RM. Transmural extent of acute myocardial infarction predicts long-term improvement in contractile function. Circulation (2001); 104: 11011107. 
Comte A, Lalande A, Walker PM, Cochet A, Legrand L, Cottin Y, Wolf J-E, Brunotte F. Visual estimation of the global myocardial extent of hyperenhancement on delayed contrast-enhanced MRI. Eur Radiol (2004); 14: 2182-2187.

Heiberg E, Engblom H, Engvall J, Hedstrom E, Ugander M, Arheden H. Semi-automatic quantification of myocardial infarction from delayed contrast enhanced magnetic resonance imaging. Scand Cardiovasc J (2005); 39: 267-275.

Heiberg E, Ugander M, Engblom H, Götberg M, Olivecrona G, Erlinge D, Arheden H. Automated quantification of myocardial infarction from MR images by accounting for partial volume effects - animal, phantom, human study. Radiology (2007); in press.

Hoffmann R, Lethen H, Marwick T, Arnese M, Fioretti P, Pingitore A, Picano E, Buck T, Erbel R, Flachskampf FA, Hanrath P. Analysis of interinstitutional observer agreement in interpretation of dobutamine stress echocardiograms. J Am Coll Cardiol (1996); 27: 330-336.

Hsu LY, Ingkanisorn WP, Kellman P, Aletras AH, Arai AE. Quantitative myocardial infarction on delayed enhancement MRI. Part II: Clinical application of an automated feature analysis and combined thresholding infarct sizing algorithm. J Magn Reson Imaging (2006a); 23: 309-314.

Hsu LY, Natanzon A, Kellman P, Hirsch GA, Aletras AH, Arai AE. Quantitative myocardial infarction on delayed enhancement MRI. Part I: Animal validation of an automated feature analysis and combined thresholding infarct sizing algorithm. J Magn Reson Imaging (2006b); 23: 298-308.

Kellman P, Chung YC, Simonetti OP, McVeigh ER, Arai AE. Multi-contrast delayed enhancement provides improved contrast between myocardial infarction and blood pool. J Magn Reson Imaging (2005); 22: 605-613.

Kim RJ, Fieno DS, Parrish TB, Harris K, Chen E-L, Simonetti O, Bundy J, Finn P, Klocke FJ, Judd RM. Relationship of MRI delayed contrast enhancement to irreversible injury, infarct age, and contractile function. Circulation (1999); 100: 1992-2002.

Kim RJ, Wu E, Rafael A, Chen EL, Parker MA, Simonetti O, Klocke FJ, Bonow R, Judd RM. The use of contrast-enhanced magnetic resonance imaging to identify reversible myocardial dysfunction. N Engl J Med (2000); 343: 1445-1453.

Klein C, Nekolla SG, Bengel FM, Momose M, Sammer A, Haas F, Schnackenburg B, Delius W, Mudra H, Wolfram D, Schwaiger M.
Assessment of myocardial viability with contrast-enhanced magnetic resonance imaging: comparison with positron emission tomography. Circulation (2002); 105: 162-167.

Miller TD, Christian TF, Hopfenspirger MR, Hodge DO, Gersh BJ, Gibbons RJ. Infarct size after acute myocardial infarction measured by quantitative tomographic $99 \mathrm{mTc}$ sestamibi imaging predicts subsequent mortality. Circulation (1995); 92: 334-341.

Moss AJ. Risk stratification and survival after myocardial infarction. N Engl J Med (1983); 309: 331-336.

Pennell DJ, Sechtem UP, Higgins CB, Manning WJ, Pohost GM, Rademakers FE, van Rossum AC, Shaw LJ, Yucel EK. Clinical indications for cardiovascular magnetic resonance (CMR): Consensus Panel report. J Cardiovasc Magn Reson (2004); 6: 727-765.

Positano V, Pingitore A, Giorgetti A, Favilli B, Santarelli MF, Landini L, Marzullo P, Lombardi M. A fast and effective method to assess myocardial necrosis by means of contrast magnetic resonance imaging. J Cardiovasc Magn Reson (2005); 7: 487-494.

Rochitte CE, Lima JA, Bluemke DA, Reeder SB, McVeigh ER, Furuta T, Becker LC, Melin JA. Magnitude and time course of microvascular obstruction and tissue injury after acute myocardial infarction. Circulation (1998); 98: 1006-1014.

Selvanayagam JB, Kardos A, Francis JM, Wiesmann F, Petersen SE, Taggart DP, Neubauer S. Value of delayed-enhancement cardiovascular magnetic resonance imaging in predicting myocardial viability after surgical revascularization. Circulation (2004); 110: 1535-1541.

Taylor AJ, Al-Saadi N, Abdel-Aty H, Schulz-Menger J, Messroghli DR, Friedrich MG. Detection of acutely impaired microvascular reperfusion after infarct angioplasty with magnetic resonance imaging. Circulation (2004); 109: 2080-2085.

Wagner A, Mahrholdt H, Holly TA, Elliott MD, Regenfus M, Parker M, Klocke FJ, Bobow RO, Kim RJ, Judd RM. Contrast-enhancement MRI and routine single photon emission computed tomography (SPECT) perfusion imaging for detection of subendocardial myocardial infarction: an imaging study. Lancet (2003); 361: 374-379.

Wu KC, Zerhouni EA, Judd RM, Lugo-Olivieri CH, Barouch LA, Schulman SP, Blumentahl RS, Lima JAO. Prognostic significance of microvascular obstruction by magnetic resonance imaging in patients with acute myocardial infarction. Circulation (1998); 97: 765-772. 